Research Paper

\title{
CTSB Knockdown Inhibits Proliferation and Tumorigenesis in HL-60 Cells
}

\author{
Sida Peng ${ }^{1,4^{*}}$, Qingqing Yang $2,4^{*}$, Huan $\mathrm{Li}^{3}$, Yuhang Pan 5 , Jiani Wang 3 , Pan $\mathrm{Hu}^{3}$, Nana Zhang $5^{凶}$ \\ 1. Department of Hematology, The First Affiliated Hospital of Guangzhou Medical University, Guangzhou, 510230, China. \\ 2. Department of Clinical Laboratory, The First Affiliated Hospital of Guangzhou Medical University, Guangzhou, 510230, China. \\ 3. Breast Cancer Center, The Third Affiliated Hospital of Sun Yat-sen University, Guangzhou, 510000, China. \\ 4. Cell genetics laboratory, The First Affiliated Hospital of Guangzhou Medical University, Guangzhou, 510230, China. \\ 5. Department of Pathology, the Third Affiliated Hospital of Sun Yat-sen University, Guangzhou510000, P. R. China. \\ * Sida Peng and Qingqing Yang contributed equally to this work.
}

$\triangle$ Corresponding author: Dr. Nana Zhang, Department of Pathology, the Third Affiliated Hospital of Sun Yat-sen University. 600 Tianhe Road, Guangzhou, Guangdong Province, 510230, P.R. China. Tel:+86-020-85253359; E-mail: zhangnn7@mail.sysu.edu.cn and Dr. Pan Hu, Breast Cancer Center, the Third Affiliated Hospital of Sun Yat-sen University. 600 Tianhe Road, Guangzhou, Guangdong Province, 510230, P.R. China. Tel:+86-020-85252154; E-mail: hupan6@mail.sysu.edu.cn.

(1) The author(s). This is an open access article distributed under the terms of the Creative Commons Attribution License (https://creativecommons.org/licenses/by/4.0/). See http://ivyspring.com/terms for full terms and conditions.

Received: 2020.10.08; Accepted: 2021.01.04; Published: 2021.01.29

\begin{abstract}
Background: Cathepsin B (CTSB) was well documented in solid tumors, up-regulated of CTSB expression is linked with progression of tumors. However, the study of CTSB in adult leukemia has not been reported. Methods: Total RNA was isolated from PBMC (peripheral blood mononuclear cell) of AML patients and healthy donors. QRT-PCR was performed to detect the expression of CTSB. The association of CTSB expression with the patients' overall survival (OS) and disease-free survival (DFS) were analyzed. Stable HL-60 CTSB-shRNA cell lines were established by retrovirus infection and puromycin selection. Cell proliferation was detected by CCK-8 analysis. Tumorigenesis ability was analyzed by soft agar and xenograft nude mice model. Western blot was performed to detect the expression of CTSB and the proteins of cell signaling pathway. Results: The mRNA expression level of CTSB was up-regulated in AML patients compared to healthy control $(p<0.001)$, and CTSB expression was significantly higher in M1, M2, M4 and M5 AML samples than healthy control. The CTSB expression in AML was associated with WBC count $(p=0.037)$. Patients with high CTSB expression had a relatively poor OS $(p=0.007)$ and a shorter DFS $(p=0.018)$. Moreover, the expression level of CTSB may act as an independent prognostic factor for both OS $(p=0.011)$ and DFS $(p=0.004)$. Knockdown CTSB expression in HL-60 cells could inhibit the cells' proliferation and tumorigeneses in vitro and in vivo. Further study showed knockdown CTSB expression in HL-60 cells could inactive the AKT signaling pathway. Conclusions: CTSB mRNA was upregulated in AML patients. CTSB overexpression was correlated with poor prognosis and may serve as an independent prognostic factor for both OS and DFS in AML patients. Knockdown CTSB expression in HL-60 cells could inhibit the cells' proliferation and tumorigenesis. The underlying mechanism may be the inhibition of the AKT signaling pathway.
\end{abstract}

Key words: CTSB; proliferation; tumorigenesis; AML; AKT pathway.

\section{Introduction}

Acute myeloid leukemia (AML) is now recognized as a hematological malignancy characterized by the blockage of myeloid differentiation and uncontrolled clonal proliferation [1]. AML, poses a significant clinical burden in the worldwide, and is the most common type of leukemia in adults with an incidence of 3.7 per 100,000 people.
Based on the newly update data of leukemia, the incidence of AML in USA is 5.2 per 100,000 for males, 3.6 per 100,000 for females [2]. Despite recent advance in the AML, the overall survival rate remains $<50 \%[3$, 4]. Further studies are required eagerly to clarify the underlying mechanisms of AML and provide novel biomarker for prognosis and treatment. In view of the 
fact that accurate genetic examinations in treatment and prognosis of AML patients [5], it is important to develop novel biomarkers related to AML.

Cathepsin B (CTSB) was primarily found as a cysteine protease that was involved in the degradation of lysosomal proteins [6]. Over expression of CTSB has been reported in numerous types of solid tumors, including salivary adenoid cystic carcinoma, brain tumor, breast tumor, colorectal tumor, and so on [7-10]. We have reported CTSB was overexpressed in gastric carcinoma [11] but down regulated in hepatocellular carcinoma [12]. It was considered as a potential biomarker of poor prognosis [7-10].

It was reported that overexpression of CTSB could promote cell invasion and metastasis of colorectal cancer, liver cancer, gastric cancer, glioma and ovarian cancer [13-17]. In addition, silence or inhibition of CTSB expression has been reported to inhibit cell proliferation, including endometrial cancer cells, meningioma cells, and human $\mathrm{T}$ cells [18-20]. Here we reported that CTSB mRNA was upregulated in AML peripheral blood samples and related to patient's poor prognosis. Then CTSB functions were investigated using the HL-60 AML cell line by knockdown the expression of CTSB. Our results showed that knockdown CTSB by shRNA could decrease cell proliferation and tumorigenesis in HL-60 cells. The analysis of pathway signals showed that knockdown CTSB in HL-60 cells could decrease the expression of $\mathrm{p}$-AKT. So decreased CTSB expression repressed the cell proliferation and tumorigenesis maybe through AKT inactivation in HL-60 cells.

\section{Materials and Methods}

\section{Patients and specimens}

Eighty seven newly diagnosed AML patients from Aug 2010 to Jun 2014 and 36 healthy control at the First Affiliated Hospital of Guangzhou Medical University were enrolled in this study. AML and healthy control peripheral blood samples were collected and mononuclear cells were isolated by Ficoll-Hypaque gradient separation according the manufacturer protocol. Characteristics of AML patients are described in Table 1. The follow-up time of the AML patients ranged from 1 to 119 months. The median follow-up time was up to 35 months. With the informed consent of the patients, the peripheral blood samples of AML patients were collected before anti-leukemia treatment. The study was approved by the Ethics Committee of the first affiliated Hospital of Guangzhou Medical University.

\section{Cell culture}

HL-60 cells (Cell bank of the Chinese Academy of Sciences, Shanghai) were cultured in RPMI-1640 medium (Hyclone, Logan, USA) supplemented with $10 \%$ fetal bovine serum (FBS; ThermoFisher Gibco, USA), penicillin (100 units/ml), and streptomycin (100 units $/ \mathrm{ml}$ ) maintained at $37{ }^{\circ} \mathrm{C}$ and $5 \% \mathrm{CO} 2$ incubator.

\section{Reverse transcription and quantitative real-time PCR assays (qRT-PCR)}

Total RNA was isolated from PBMC (peripheral blood mononuclear cell) of AML patients using TRIzol reagent (Transgen Biotech, Beijing, China), according to the protocol of manufacturer. First Strand cDNA Synthesis Kit (Thermo Fisher scientific, Waltham, MA, USA) was used to reverse transcribe RNA to cDNA according to the manufacturer's protocol. A total of $1.0 \mathrm{ug}$ of mRNA was used to reverse transcribe RNA to cDNA. RT-qPCR was carried out with SYBR Green Master Mix (Transgen Biotech, Beijing, China). The qPCR was performed at a total $10 \mu \mathrm{l}$ and the following thermocycling conditions were used for the qPCR: Initial denaturation at $95^{\circ} \mathrm{C}$ for $10 \mathrm{~min} ; 40$ cycles of $95^{\circ} \mathrm{C} 15 \mathrm{sec}$ and $55^{\circ} \mathrm{C}$ for $20 \mathrm{sec}$, followed by a dissociation step at $95{ }^{\circ} \mathrm{C}$ for $15 \mathrm{~s}$, and $60^{\circ} \mathrm{C}$ for $30 \mathrm{~s}$, Primer sequences were used as follows: CTSB sense 5'-GCAGGCCGGGCACAAC-3', antisense 5'-GGAGGCCCAGAGCTGCCAC-AT-3'. GAPDH sense 5'-TGTTGCCATCAATGACC-CC-3', antisense 5'-CTCCACGACGTACTCAGC-3'. The results were normalized to the expression of GAPDH.

All experiments were performed in triplicate. The relative expression of target gene was calculated by $2-\Delta \mathrm{Ct}$ method $(\Delta \mathrm{Ct}=\mathrm{CT}$ target gene-CTGAPDH).

\section{Western blot analysis}

Western blot was performed as previously described [21]. The blots were probed with rabbit anti-CTSB antibody (Proteintech Group, Inc., Rosemont, IL, USA), anti-AKT antibody, anti-p-AKT antibody, anti-p-ERK antibody, anti-p38 antibody, anti-p-p38 antibody (Cell signaling, Billerica, MA). Mouse anti-GAPDH antibody (Cell signaling, Billerica, MA) was used as loading control.

\section{Anchorage-independent cell growth}

The ability of CTSB-knockdown HL-60 cell lines growing in anchorage-independence was assessed by colony formation in soft agar as previously described [22]. 5000 cells were suspended in $1 \mathrm{ml}$ of RPMI 1640 with $0.33 \%$ agarose (Sigma), 10\% FBS and plated into a well of 12-well plate. After 14 days culture, colonies were identified with a diameter $>125 \mu \mathrm{m}$. 


\section{Short hairpin RNA (shRNA) constructs and retroviral infection}

Stable knockdown of endogenous CTSB was performed using retrovirus constructs targeting CTSB. The shRNA target sequences were: shRNA1, 5-CCAACACGTCAC CGGAGAGAT-3; shRNA2, 5-GCTGGTCAACTATGTCAACAA-3. The synthetic oligos were cloned into the pSuper-retro-puro vector. The successful plasmid construction was verified by DNA sequencing. Production of retrovirus was performed according to the instructions in 293FT cells. HL-60 cells were subjected with infection of retrovirus expressing CTSB-shRNA1 and CTSB-shRNA2. An empty pSuper-retro-puro was used as a control (Scramble).

\section{Cell proliferation assay}

HL-60 CTSB-shRNA1 cells, HL-60 CTSBshRNA2 cells and HL-60 Scramble cells were seeded in a 96-well plate at a density of 2000 cells/well and maintained at $37^{\circ} \mathrm{C}$ in $5 \% \mathrm{CO} 2$ incubator for $24 \mathrm{~h}, 48 \mathrm{~h}$, 72h, $96 \mathrm{~h}$ and 120h. $10 \mu \mathrm{l}$ of the Cell Counting Kit-8 (CCK8, Transgen Biotech, Beijing, China) was added per well and the plate was incubated for $2 \mathrm{~h}$ at $37^{\circ} \mathrm{C}$ in $5 \% \mathrm{CO} 2$ incubator, the absorbance at $450 \mathrm{~nm}$ was measured on a microplate reader.

\section{Mice}

The tumorigenic capacity was analyzed by subcutaneous injecting HL-60 Scramble cells and HL-60 CTSB-shRNA2 cells into nude mice. All the mice were bred and maintained under SPF conditions in the Department of Animal Center, the first Affiliated Hospital of Guangzhou Medical University. Animal protocol was approved by the Institutional Animal Care and Use Committee of Guangzhou Medical University. 12 healthy nude mice, which were four to six weeks old, were randomly assigned to each group. Each mouse was subcutaneous injected with $5 \times 10^{6}$ cells in 1:1 serum free medium and Matrigel solution. Tumor growth was measured and tumor volume was calculated according to the formula: tumor volume $[\mathrm{mm} 3]=($ length $[\mathrm{mm}]) \times$ $(\text { width }[\mathrm{mm}])^{2} \times 0.5[23]$.

\section{Statistical analysis}

Statistical analyses were performed using Statistical Program for Social Sciences 20.0 (SPSS, Chicago, IL, USA) and Graph Pad Prism 5.0 (Graph Pad Software, La Jolla, CA, USA). The correlation between the CTSB expression and the clinicopathological parameters of patients with AML was assessed by a chi-square test. The Kaplan-Meier analysis was used to evaluate the survival curve of distinct CTSB expression of AML patients. The prognostic value of CTSB on AML patients' survival was analyzed by the Cox proportional hazard model. A p-value less than 0.05 was regarded as statistically significant.

\section{Result}

\section{CTSB was up-regulated in AML and correlated with clinical outcomes.}

CTSB mRNA expression levels were evaluated in the peripheral blood samples of 87 newly diagnosed AML patients and 36 healthy control by qRT-PCR methods. A statistically difference was found in the mRNA expression level of CTSB between newly diagnosed AML patients and healthy control samples $(p<0.001$, Figure 1A). The mRNA expression of CTSB in AML patients exhibited obviously higher level (median value 0.039, range: 0.005-0.129) than healthy controls (median value 0.021, range: 0.001-0.055). We also analyzed the expression of CTSB between healthy control and FAB category of AML. CTSB expression was significantly higher in M1, M2, M4 and M5 AML samples than healthy control (Figure 1B). Moreover, correlation of CTSB expression with clinical characteristics of AML patients was analyzed. The patient samples were divided into two groups according to the expression of CTSB gene (high vs. low). High CTSB expression was detected in 36 samples $(41.38 \%)$ and low CTSB expression was detected in 51 samples $(58.62 \%$, Table 1$)$. The relationship between CTSB expression and other clinical indexes was analyzed. We found the expression of CTSB was associated with WBC count $(p=0.037)$. However, there were no significant correlations between CTSB expression with patients' gender, age, FAB subtype, HGB, PLT, blast in BM and complete remission (Table 1 ).

\section{CTSB expression was correlated with OS and DFS}

Furthermore, we explored the association between the expression of CTSB and the AML patients' clinical outcomes. The data showed patients with a high expression level of CTSB had a poor overall survival (OS) $(p=0.007)$ and a short disease-free survival (DFS) $(p=0.018)$ (Figure 2A, B). Univariate Cox proportional hazard regression analysis showed that both blast in BM $(p=0.001)$ and the CTSB expression level $(p=0.010)$ were prognostic factors for OS (Table 2). The patients' age $(p=0.003)$, blast in BM $(p<0.001)$ and CTSB expression level $(p=0.021)$ were associated with DFS (Table 3$)$. The multivariate adjustment for the above significant clinicopathological features showed that the expression level of CTSB was an independent 
prognostic factor for both OS (Hazard ratio [HR] $=0.393,95 \%$ confidence interval [CI], 0.192-0.805, $p=0.011$ ) and DFS (Hazard ratio $[\mathrm{HR}]=0.363,95 \%$ confidence interval [CI], 0.182-0.725, $p=0.004$ ) of AML patients.

\section{CTSB knockdown inhibits the proliferation and tumorigenesis of HL-60 cells}

HL-60 cells were infected with CTSB-shRNA1, CTSB-shRNA2 retrovirus which were mediated by pSuper-retro-puro vector. Western blot analysis was used to determine the effect of CTSB knockdown. The result showed that the CTSB protein expression level was decreased in CTSB-shRNA cells (Fig. 2A). CCK-8 analysis was performed to detect the cell proliferation effect of CTSB-shRNA in HL-60 cells. As show in Figure 2B, a significant decrease in proliferation rate was observed in CTSB-shRNA1 and CTSB-shRNA2 cells compared to scramble cells, indicating that CTSB knockdown could inhibit HL-60 cells' proliferation (Fig. 2B). To further examine tumor-suppressing activity of CTSB-shRNA, softagar assay was performed to examine the anchorage-independent growth of HL-60 cells. The results showed that CTSB-shRNA could inhibit anchorage-independent growth of HL-60 cells (Fig. 2C, D).

\section{CTSB knockdown attenuates tumor growth of HL-60 cells in nude mice model}

The effect of CTSB knockdown on tumor growth in vivo was examined in a xenograft nude mice model (Fig. 3). Mice were injected subcutaneously with $5 \times 10^{6}$ individual cells infected of CTSB-shRNA1 and scramble retrovirus. Then the tumor size and weight generated by CTSB-shRNA HL-60 cells and scramble cells were compared. The results showed a smaller tumor volume and less tumor weight in CTSB-shRNA group than scramble group (Fig. 3B, C). Thus, CTSB knockdown attenuates tumor growth of HL-60 cells in vivo.

\section{CTSB may regulate HL-60 cell growth and tumorigenesis via regulation of AKT signaling pathway}

To investigate the underlying mechanisms of decreased cell proliferation and tumorigenesis of HL-60 CTSB-shRNA cells, western blot analysis was performed to detect the common tumor-related signaling pathways. We analyzed the MAPK/P38 pathway, the AKT pathway, and the ERK pathway. Then we found that when we knockdown the expression of CTSB in HL-60 cells, the p-AKT expression was downregulated, but there was no significant difference on the expressions of p-p38 and p-ERK between HL-60 shRNA cells and scramble control cells (Fig. 4). So CTSB may regulate HL-60 AML cell growth and tumorigenesis via the regulation of AKT signaling pathway.

Table 1. Correlation of CTSB expression with clinicopathologic features

\begin{tabular}{|c|c|c|c|c|}
\hline \multirow[t]{2}{*}{ Characteristics } & \multirow[t]{2}{*}{ Total $(n=87)$} & \multicolumn{2}{|l|}{ CTSB } & \multirow[t]{2}{*}{$P$ value } \\
\hline & & $\operatorname{Low}(\mathrm{n}=51)$ & High $(n=36)$ & \\
\hline Gender & & & & 0.810 \\
\hline Male & $47(54.0 \%)$ & $27(57.4 \%)$ & $20(42.6 \%)$ & \\
\hline Female & $40(46.0 \%)$ & $24(60.0 \%)$ & $16(40.0 \%)$ & \\
\hline Age(years) & & & & 0.454 \\
\hline$\leq 60$ & $64(73.6 \%)$ & $36(56.2 \%)$ & $28(43.8 \%)$ & \\
\hline$>60$ & $23(26.4 \%)$ & $15(65.2 \%)$ & $8(34.8 \%)$ & \\
\hline FAB subtype & & & & 0.953 \\
\hline M1 & $15(17.2 \%)$ & $8(53.3 \%)$ & $7(46.7 \%)$ & \\
\hline M 2 & $22(25.3 \%)$ & $12(54.5 \%)$ & $10(45.5 \%)$ & \\
\hline M3 & $9(10.3 \%)$ & $6(66.7 \%)$ & $3(33.3 \%)$ & \\
\hline M 4 & $12(13.8 \%)$ & $7(58.3 \%)$ & $5(41.7 \%)$ & \\
\hline M 5 & $29(33.3 \%)$ & $18(62.1 \%)$ & $11(37.9 \%)$ & \\
\hline $\mathrm{WBC}\left(\times 10^{9} / \mathrm{L}\right)$ & & & & 0.037 \\
\hline$<10$ & $44(50.6 \%)$ & $28(63.6 \%)$ & $16(36.4 \%)$ & \\
\hline$\geq 10$ & $43(49.4 \%)$ & $23(53.5 \%)$ & $20(46.5 \%)$ & \\
\hline $\mathrm{HGB}(\mathrm{g} / \mathrm{L})$ & & & & 0.988 \\
\hline$<80$ & $46(52.9 \%)$ & $27(58.7 \%)$ & $19(41.3 \%)$ & \\
\hline$\geq 80$ & $41(47.1 \%)$ & $24(58.5 \%)$ & $17(41.5 \%)$ & \\
\hline $\operatorname{PLT}\left(\times 10^{9} / \mathrm{L}\right)$ & & & & 0.196 \\
\hline$<10$ & $46(52.9 \%)$ & $24(52.2 \%)$ & $22(47.8 \%)$ & \\
\hline$\geq 10$ & $41(47.1 \%)$ & $27(65.9 \%)$ & $14(34.1 \%)$ & \\
\hline Blast in BM & & & & 0.928 \\
\hline$<50 \%$ & $43(49.4 \%)$ & $25(58.1 \%)$ & $18(41.9 \%)$ & \\
\hline$\geq 50 \%$ & $44(50.6 \%)$ & $26(59.1 \%)$ & $18(40.9 \%)$ & \\
\hline Complete Remission & & & & 0.498 \\
\hline Yes & $40(46.0 \%)$ & $25(62.5 \%)$ & $15(37.5 \%)$ & \\
\hline No & $47(54.0 \%)$ & $26(55.3 \%)$ & $21(44.7 \%)$ & \\
\hline
\end{tabular}

Table 2. Cox-regression analysis of parameters associated with Overall survival of AML patients

\begin{tabular}{lllll}
\hline Factor & \multicolumn{3}{l}{ Univariate } & \multicolumn{3}{l}{ Multivariate } \\
\cline { 2 - 5 } & HR $(95 \% \mathrm{CI})$ & $P$ value & HR(95\%CI) & $P$ value \\
\hline Blast in BM & & & Reference & \\
$<50 \%$ & Reference & & & \\
$\geq 50 \%$ & $0.244(0.110-0.543)$ & 0.001 & $0.245(0.110-0.545)$ & 0.001 \\
CTSB expression & & & & \\
Low & Reference & & Reference & \\
High & $0.390(0.191-0.795)$ & 0.010 & $0.393(0.192-0.805)$ & 0.011 \\
\hline
\end{tabular}

HR: hazard ratio. $95 \% \mathrm{CI}$ : $95 \%$ confidence interval

Table 3. Cox-regression analysis of parameters associated with Disease free survival of AML patients

\begin{tabular}{lllll}
\hline Factor & Univariate & \multicolumn{3}{c}{ Multivariate } \\
\cline { 2 - 5 } & HR(95\%CI) & $P$ value & HR(95\%CI $)$ & $P$ value \\
\hline Age(years) & Reference & & Reference & \\
$\leq 60$ & $0.398(0.218-0.730)$ & 0.003 & $0.366(0.194-0.690)$ & 0.002 \\
$>60$ & & & & \\
Blast in BM & Reference & & Reference & \\
$<50 \%$ & $0.246(0.124-0.491)$ & 0.000 & $0.261(0.131-0.521)$ & 0.000 \\
$\geq 50 \%$ & Reference & & Reference & \\
CTSB expression & & & \\
Low & $0.465(0.242-0.893)$ & 0.021 & $0.363(0.182-0.725)$ & 0.004 \\
High & & & & \\
\hline
\end{tabular}


A

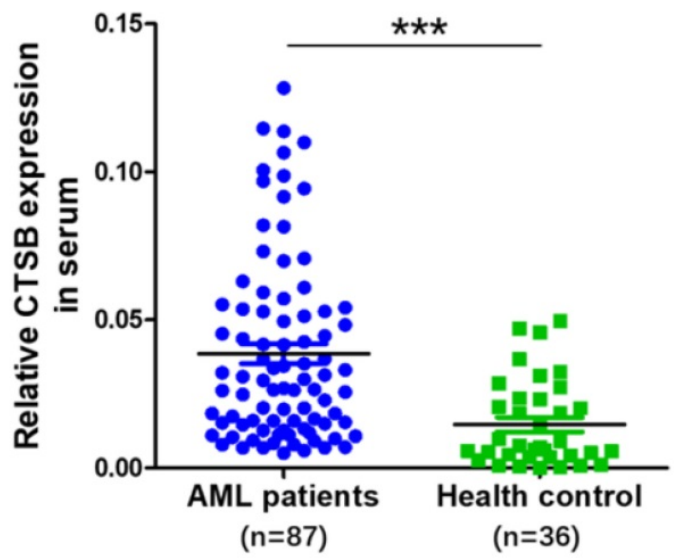

C

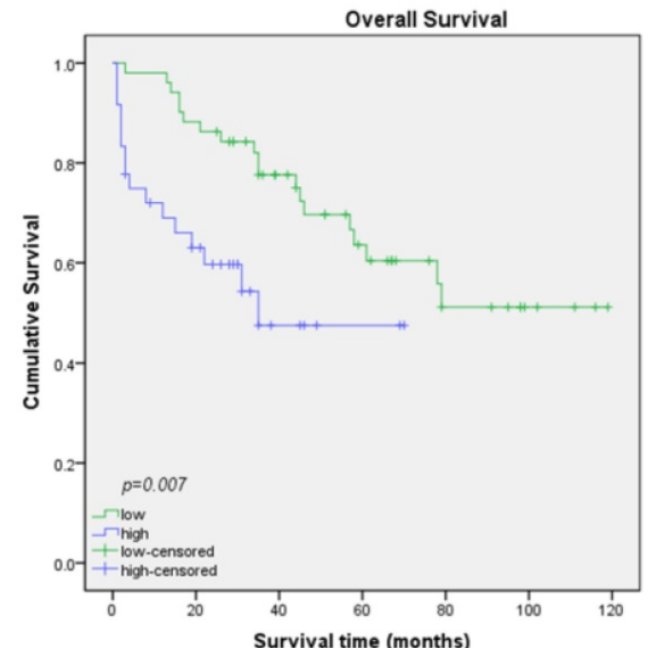

B

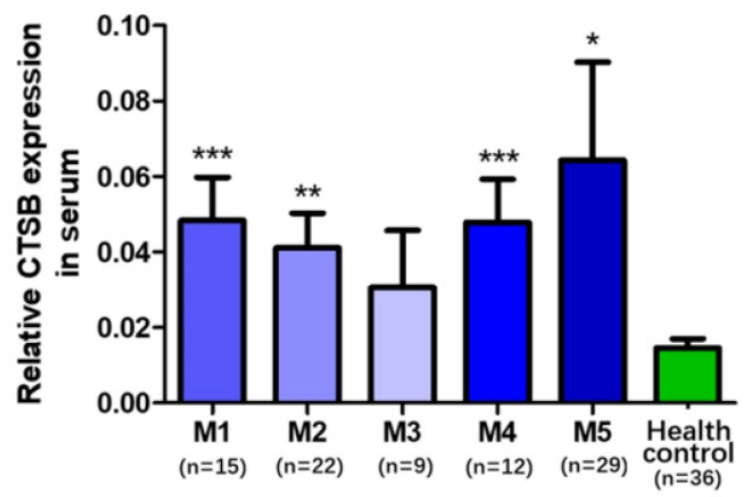

D

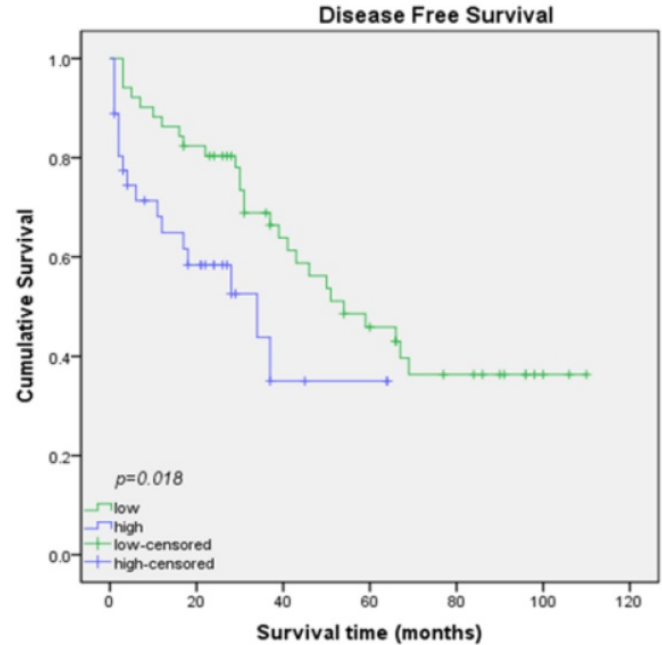

Figure 1. CTSB expression was up-regulated in AML patients and related to patients' poor OS and short DFS. (A) Expression levels of CTSB in AML patients (median value 0.039 , range: $0.005-0.129$ ) and healthy controls (median value 0.021 , range: $0.001-0.055$ ). The expression of CTSB was upregulated in AML compared with healthy controls ( $p<0.001$ ); (B) Expression levels of CTSB in FAB category of AML patients showed that CTSB expression was significantly higher in M1, M2, M4 and M5 AML samples than healthy control; (C) The OS rate of AML patients with high and low CTSB expression ( $p=0.007)$; The P-value was calculated using the log-rank test. (D) The DFS rate of AML patients with high or low CTSB expression $(p=0.018)$. The $P$-value was calculated using the log-rank test.

A

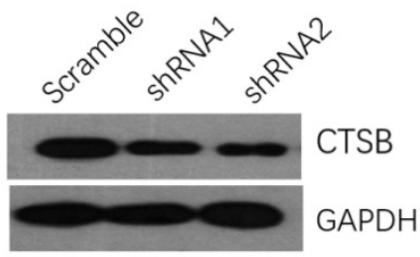

B

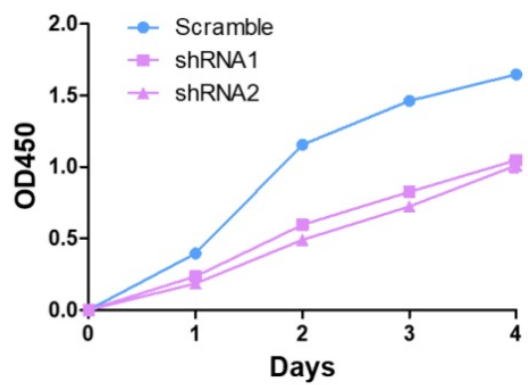

C

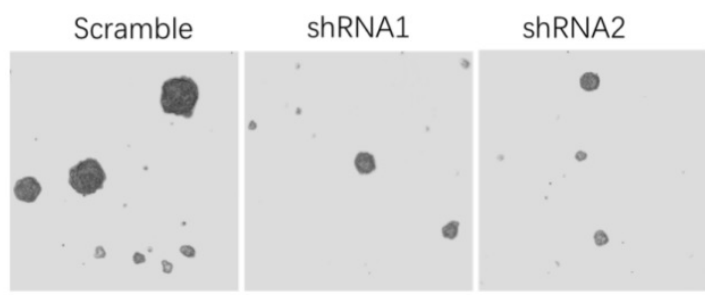

D

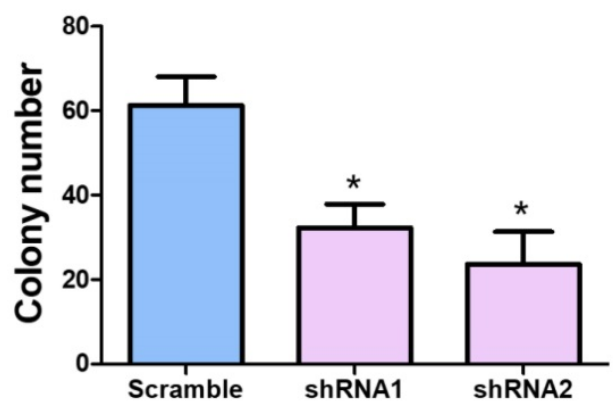

Figure 2. Knockdown CTSB expression in HL-60 cells inhibit cell proliferation and colony formation in vitro. (A) CTSB expression levels were detected by western blotting in HL-60 CTSB-shRNA cells and scramble cells; (B) CCK assay was performed. Decreased proliferation of HL-60 cells was detected after CTSB knockdown. (C, D) Softagar assay was performed to examine the anchorage-independent growth of HL-60 cells. Decreased colony formation ability was observed in HL-60 cells after CTSB knockdown (shRNA1 $p=0.029$, shRNA2 $p=0.021$ ). 
A

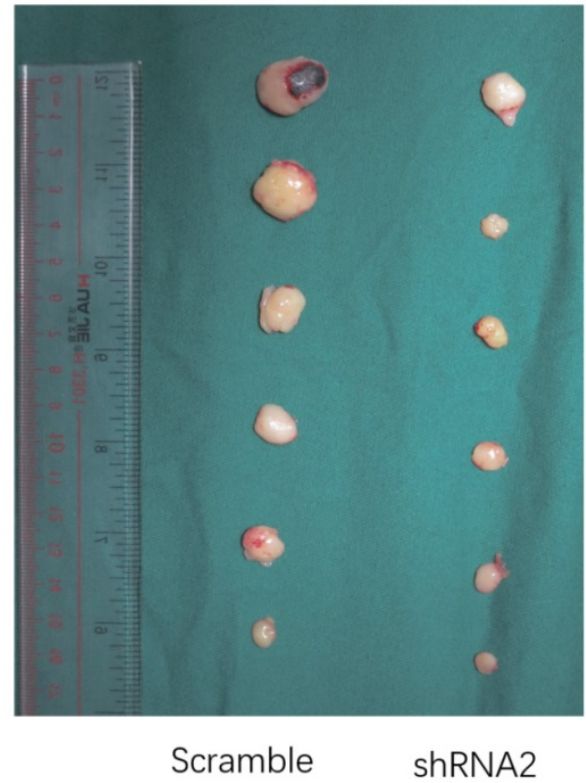

B

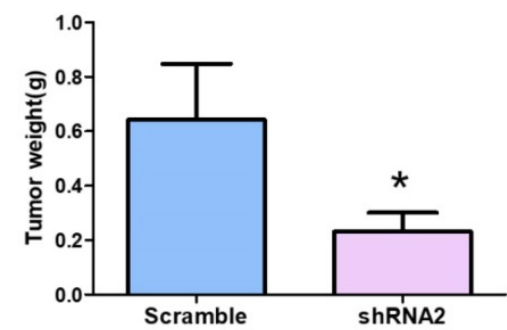

C

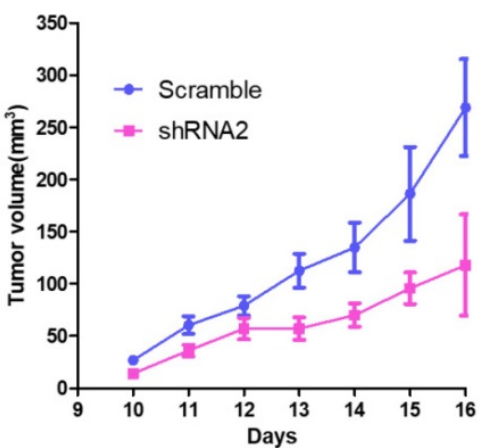

Figure 3. CTSB knockdown attenuates tumor growth of HL-60 cells in nude mice model. (A) HL-60 CTSB-shRNA2 and scramble cells were injected subcutaneously to generated tumors in mice. All mice were executive 3 weeks after injection; (B) Tumor size was measured. The result showed that tumor volume of CTSB-shRNA2 was decreased than scramble mice. (C) Tumor weight showed a decrease in CTSB-shRNA mice $(p=0.033)$.

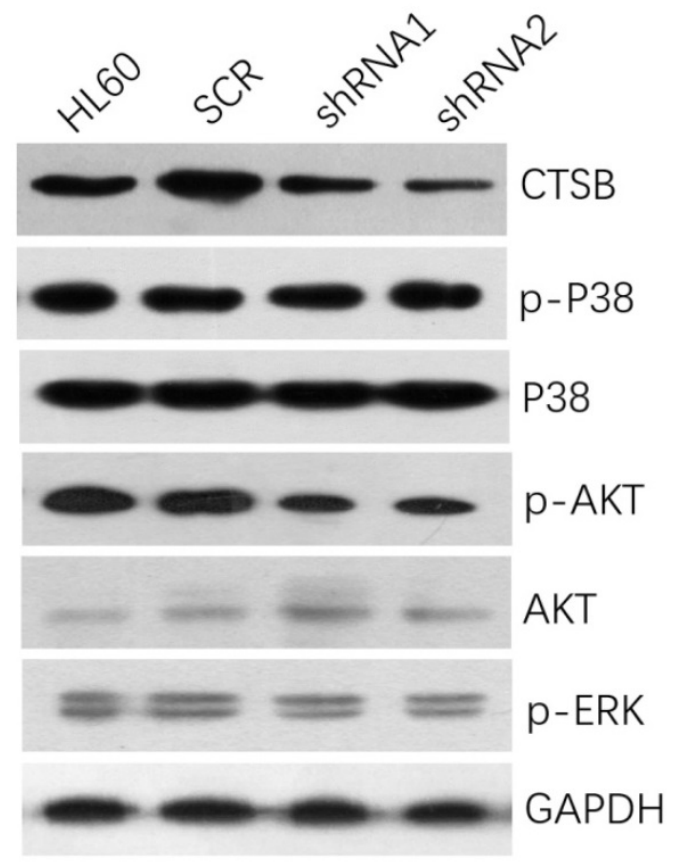

Figure 4. CTSB knockdown could inhibit the expression of p-AKT. Protein levels of p-p38, p-Akt, p-ERK were assessed in HL-60 CTSB-shRNA cells and scramble cells by western blotting, the $\mathrm{P}$-AKT expression was downregulated after CTSB knockdown.

\section{Discussion}

Cathepsin is a lysosomal protease belonging to papain family [24]. Cathepsins can be classified into serine cathepsins, cysteine cathepsins and aspartate cathepsins[25]. Human cysteine cathepsins are essential in the degradation of the proteins that are internalized in the lysosomes [26]. However, cathepsins were reported not only localized endoly-sosomal compartment, they also localized in cell cytoplasm, nucleus, mitochondria and extracellular. This indicated cathepsins may have broad biological activity [27, 28]. CTSB, a kind of lysosomal cysteine protease, has been reported to be a potentially effective biomarker as well as an important contributor to the progression of different types of cancers.

It was reported that CTSB might be an optimal diagnostic and prognostic marker [29-31], and CTSB can be developed to design enhanced drug delivery approaches in the recent years [32]. We also reported that CTSB may serve as an optical prognostic marker in gastric carcinoma and hepatocellular carcinoma $[11,12]$. Many researchers presented that CTSB acted as a metastasis-related gene $[7,13,18]$. The plasma membrane localization of CTSB correlated with metastatic tumors [33, 34]. CTSB was also reported related to autophagy. Autophagy, a lysosomal-dependent process could prevent cancer by removing damaged proteins and reducing reactive oxygen species (ROS), and promoting the cell death. CTSB inhibition was showed to increase cell autophagy, and accumulation autophagic biomarkers could be observed $[35,36]$.

In this study, we found the expression of CTSB in AML patients was up-regulated compared to health control group. Patients with high expression of 
CTSB displayed a poor OS and DFS. Cox regression analysis showed CTSB mRNA expression was an independent prognostic factor for AML patients' OS and DFS. This result indicates the CTSB expression may act as a predictor for AML patients' prognosis and survival. Misti Jain, etc. reported that both CTSB and CTSL mRNA expression levels were up-regulated in pediatric acute myeloid leukemia [36]. Total 24 pediatric AML patients were enrolled in the analysis. The result showed pediatric AML patients with higher CTSB expression exhibited an inferior DFS and OS compared to lower CTSB expression patients [36]. This is the first report that CTSB was up-regulated in pediatric AML. Our report addressed CTSB expression and its clinical significance in adult AML patients. CTSB expression was up-regulated and correlated with poor prognosis of adult AML patients.

To clarify the tumor-associated functions and related mechanism of CTSB gene in AML. We generated HL-60 CTSB-shRNAs cell lines using retrovirus infection. Based on the cell model, we observed knockdown CTSB expression could result in inhibition of HL-60 cells' proliferation. Anchorageindependent cell growth analysis showed that CTSB-shRNA could inhibit anchorage-independent growth of HL-60 cells in vitro. Furthermore, HL-60 CTSB-shRNA cells were subcutaneously injected in mice to generated tumors. Inhibition of tumor weight and tumor volume by CTSB-shRNA was observed in vivo. However, we did not observe a significant change in the invasiveness of HL-60 CTSB-shRNA cells compared to control cells (data was not shown). We have not performed the cell autophagic study about CTSB in AML. It is an interesting project and we will clarify it in the next report.

At last, we assessed the AKT, p38, ERK oncogenic cell signaling pathway protein levels to found which cell signaling pathway enrolled in CTSB regulating. We found that p-AKT levels were significantly decreased after CTSB was knockdown in HL-60 cells. These findings suggested that knockdown CTSB might inhibit HL-60 cell proliferation and tumorigenesis via the inactivation of AKT signaling pathway. However, this mechanism study was simple and superficial. It only provided knockdown CTSB may inhibit HL-60 cells' proliferation and tumorigenesis through inactivation AKT signaling pathway, but we don't know how p-AKT was downregulated and AKT pathway was inactivated by CTSB-shRNA, and there is no clue if there are any other mechanism contribute to CTSB-shRNA inducing inhibition of cells' proliferation and tumorigenesis. All of these need more experiments to clarify in the future.

\section{Conclusions}

In summary, CTSB expression was up-regulated in AML patients compare with normal control. Most importantly, patients with high CTSB expression have a poor OS and short DFS. Knockdown CTSB expression could inhibit cell proliferation and tumorigenesis in HL-60 AML cell line. The underlying mechanism may through regulating AKT oncogenic cell signaling pathway.

\section{Abbreviations}

AML: acute myeloid leukemia; CTSB: Cathepsin B; PBMC: peripheral blood mononuclear cell; OS: overall survival; DFS: disease-free survival.

\section{Acknowledgments}

We thank the funding support of National Natural Science Foundation of China, Guangdong Province Natural Science Foundation and Guangzhou Science and Technology Project.

\section{Funding}

This study was supported by grants from the National Natural Science Foundation of China (81902416) and the Natural Science Foundation of Guangdong Province, China (2017A030313858, 2018A0303130324).

\section{Availability of data and materials}

The datasets and materials used in the current study are available from the corresponding authors.

\section{Ethics approval and consent to participate}

The Committee on the Ethics of the First Affiliated Hospital of Guangzhou Medical University approved this study. All the patients and healthy control volunteers provided written informed consent for the use of clinical specimens. Animals were purchased from Animal experimental center, Shanghai, China. The protocol was approved by the Institutional Animal Care and Use Committee of Sun Yat-sen University. All mouse procedures were performed in strict accordance with the recommendations in the Guide for the Care and Use of Laboratory Animals of Sun Yat-sen University. Animal experiment was performed in Sun Yat-sen University and all surgery was performed under sodium pentobarbital anesthesia, and all efforts were made to minimize suffering.

\section{Authors' contributions}

Huan Li and Qingqing Yang designed this study. Yuhang Pan and Sida Peng carried out the in vitro study and drafted the manuscript. Nana Zhang and Pan Hu carried out the statistical analysis and assisted 
with the manuscript preparation and data analysis. Jiani Wang carried out the in vivo study. All authors read and approved the final manuscript.

\section{Competing Interests}

The authors have declared that no competing interest exists.

\section{References}

1. Ferrara F, Schiffer CA. Acute myeloid leukaemia in adults. Lancet. 2013; 381: 484-95.

2. [Internet] 2018-2019 F. Cancer Facts and Statistics. https://wwwcancerorg/research/cancer-facts-statisticshtml 2019.

3. Watts J, Nimer S. Recent advances in the understanding and treatment of acute myeloid leukemia. F1000Res. 2018; 7.

4. Winer ES, Stone RM. Novel therapy in Acute myeloid leukemia (AML): moving toward targeted approaches. Ther Adv Hematol. 2019; 10: 2040620719860645

5. Dohner H, Estey E, Grimwade D, Amadori S, Appelbaum FR, Buchner T, et al. Diagnosis and management of AML in adults: 2017 ELN recommendations from an international expert panel. Blood. 2017; 129: 424-47.

6. Ferri KF, Kroemer G. Organelle-specific initiation of cell death pathways. Nat Cell Biol. 2001; 3: E255-63.

7. Wu JS, Li ZF, Wang HF, Yu XH, Pang X, Wu JB, et al. Cathepsin B defines leader cells during the collective invasion of salivary adenoid cystic carcinoma. Int J Oncol. 2019; 54: 1233-44.

8. Yano M, Hirai K, Naito Z, Yokoyama M, Ishiwata T, Shiraki $Y$, et al. Expression of cathepsin B and cystatin $\mathrm{C}$ in human breast cancer. Surg Today. 2001; 31: 385-9.

9. Strojnik T, Kos J, Zidanik B, Golouh R, Lah T. Cathepsin B immunohistochemical staining in tumor and endothelial cells is a new prognostic factor for survival in patients with brain tumors. Clin Cancer Res. 1999; 5: 559-67.

10. Kos J, Nielsen HJ, Krasovec M, Christensen IJ, Cimerman N, Stephens RW, et al. Prognostic values of cathepsin B and carcinoembryonic antigen in sera of patients with colorectal cancer. Clin Cancer Res. 1998; 4: 1511-6.

11. Lihua Xu SP, Nana Zhang, Ruiming Liu, Qunai Huang, Xi Li, Jiani Wang. Expression status of cathepsin B may as a prognostic marker for human gastric carcinoma. Int J Clin Exp Pathol. 2016; 9: 6988-98.

12. Lingyu Qin JC, Jiani Wang, Jingmei Ye, Huo Tan, Lihua Xu. Expression of cathepsin B in human hepatocellular carcinoma and its prognostic significance. Int J Clin Exp Pathol. 2016; 9: 1343-50.

13. Bian B, Mongrain S, Cagnol S, Langlois MJ, Boulanger J, Bernatchez G, et al. Cathepsin B promotes colorectal tumorigenesis, cell invasion, and metastasis. Mol Carcinog. 2016; 55: 671-87.

14. Chen WN, Chen JY, Jiao BY, Lin WS, Wu YL, Liu LL, et al. Interaction of the hepatitis B spliced protein with cathepsin B promotes hepatoma cell migration and invasion. J Virol. 2012; 86: 13533-41.

15. Rempel SA, Rosenblum ML, Mikkelsen T, Yan PS, Ellis KD, Golembieski WA, et al. Cathepsin B expression and localization in glioma progression and invasion. Cancer Res. 1994; 54: 6027-31.

16. Liu Y, Xiao S, Shi Y, Wang L, Ren W, Sloane BF. Cathepsin B on invasion and metastasis of gastric carcinoma. Chin Med J (Engl). 1998; 111: 784-8.

17. Nishikawa H, Ozaki Y, Nakanishi T, Blomgren K, Tada T, Arakawa A, et al. The role of cathepsin B and cystatin $C$ in the mechanisms of invasion by ovarian cancer. Gynecol Oncol. 2004; 92: 881-6.

18. Bao W, Fan Q, Luo X, Cheng WW, Wang YD, Li ZN, et al. Silencing of Cathepsin $B$ suppresses the proliferation and invasion of endometrial cancer. Oncol Rep. 2013; 30: 723-30.

19. Gogineni VR, Gupta R, Nalla AK, Velpula KK, Rao JS. uPAR and cathepsin B shRNA impedes TGF-beta1-driven proliferation and invasion of meningioma cells in a XIAP-dependent pathway. Cell Death Dis. 2012; 3: e439.

20. Rajah T, Chow SC. Suppression of Human T Cell Proliferation Mediated by the Cathepsin B Inhibitor, z-FA-FMK Is Due to Oxidative Stress. PLoS One. 2015; 10: e0123711.

21. Luo XD, Yang SJ, Wang JN, Tan L, Liu D, Wang YY, et al. Downregulation of SATB1 increases the invasiveness of Jurkat cell via activation of the WNT/beta-catenin signaling pathway in vitro. Tumour Biol. 2016; 37: 7413-9.

22. Rahmani M, Dai $Y$, Grant $S$. The histone deacetylase inhibitor sodium butyrate interacts synergistically with phorbol myristate acetate (PMA) to induce mitochondrial damage and apoptosis in human myeloid leukemia cells through a tumor necrosis factor-alpha-mediated process. Exp Cell Res. 2002; 277: 31-47.

23. Salom F, Piedra W, Burgos H. Tumor growth rate of pancreatic serous cystadenomas: Endosonographic follow-up with volume measurement to predict cyst enlargement. Pancreatology. 2019; 19: 122-6.

24. Turk V, Stoka V, Vasiljeva O, Renko M, Sun T, Turk B, et al. Cysteine cathepsins: from structure, function and regulation to new frontiers. Biochim Biophys Acta. 2012; 1824: 68-88
25. Rudzinska M, Parodi A, Soond SM, Vinarov AZ, Korolev DO, Morozov AO, et al. The Role of Cysteine Cathepsins in Cancer Progression and Drug Resistance. Int J Mol Sci. 2019; 20.

26. Pu J, Guardia CM, Keren-Kaplan T, Bonifacino JS. Mechanisms and functions of lysosome positioning. J Cell Sci. 2016; 129: 4329-39.

27. Cheng XW, Shi GP, Kuzuya M, Sasaki T, Okumura K, Murohara T. Role for cysteine protease cathepsins in heart disease: focus on biology and mechanisms with clinical implication. Circulation. 2012; 125: 1551-62.

28. Sever S, Altintas MM, Nankoe SR, Moller CC, Ko D, Wei C, et al. Proteolytic processing of dynamin by cytoplasmic cathepsin $\mathrm{L}$ is a mechanism for proteinuric kidney disease. J Clin Invest. 2007; 117: 2095-104.

29. Abdulla MH, Valli-Mohammed MA, Al-Khayal K, Al Shkieh A, Zubaidi A, Ahmad R, et al. Cathepsin B expression in colorectal cancer in a Middle East population: Potential value as a tumor biomarker for late disease stages. Oncol Rep. 2017; 37: 3175-80

30. Yan Y, Zhou K, Wang L, Wang F, Chen X, Fan Q. Clinical significance of serum cathepsin $\mathrm{B}$ and cystatin $\mathrm{C}$ levels and their ratio in the prognosis of patients with esophageal cancer. Onco Targets Ther. 2017; 10: 1947-54.

31. Ruan J, Zheng H, Rong X, Zhang J, Fang W, Zhao P, et al. Over-expression of cathepsin B in hepatocellular carcinomas predicts poor prognosis of HCC patients. Mol Cancer. 2016; 15: 17.

32. Ben-Nun Y, Fichman G, Adler-Abramovich L, Turk B, Gazit E, Blum G. Cathepsin nanofiber substrates as potential agents for targeted drug delivery. $\mathrm{J}$ Control Release. 2017; 257: 60-7.

33. Sloane BF, Rozhin J, Johnson K, Taylor H, Crissman JD, Honn KV. Cathepsin B: association with plasma membrane in metastatic tumors. Proc Natl Acad Sci U S A. 1986; 83: 2483-7.

34. Wendt W, Schulten $\mathrm{R}$, Stichel CC, Lubbert $\mathrm{H}$. Intra- versus extracellular effects of microglia-derived cysteine proteases in a conditioned medium transfer model. J Neurochem. 2009; 110: 1931-41.

35. Cartledge DM, Colella R, Glazewski L, Lu G, Mason RW. Inhibitors of cathepsins $\mathrm{B}$ and $\mathrm{L}$ induce autophagy and cell death in neuroblastoma cells. Invest New Drugs. 2013; 31: 20-9.

36. Cartledge DM, Colella R, Glazewski L, Lu G, Mason RW. Inhibitors of cathepsins B and L induce autophagy and cell death in neuroblastoma cells. Invest New Drugs. 2010; 31: 20-9. 